Supporting Information for

\title{
Excited State Potentials and Ligand Force-field of a Blue Copper Protein Plastocyanin
}

\author{
Koji Ando \\ School of Chemistry, University of Birmingham, \\ Edgbaston, Birmingham, B15 2TT, United Kingdom \\ E-mail: K.Ando@bham.ac.uk
}

Table S2. (Cu-Cys)-ligand Electrostatic interaction (in $\mathrm{eV}$ ).

\begin{tabular}{lrrrrrrrrr}
\hline & \multicolumn{3}{c}{$\mathrm{Cu}$} & & \multicolumn{3}{c}{ Cys84 } & \\
\cline { 2 - 3 } \cline { 7 - 8 } state & His37 & His87 & Met92 & & His37 & His87 & Met92 & total \\
\hline 0 & -3.568 & -3.478 & -0.327 & & 0.452 & 0.474 & 0.065 & -6.384 \\
1 & -3.706 & -3.611 & -0.337 & & 0.498 & 0.523 & 0.071 & -6.564 \\
2 & -3.736 & -3.641 & -0.340 & & 0.509 & 0.533 & 0.074 & -6.607 \\
3 & -3.733 & -3.638 & -0.340 & & 0.506 & 0.531 & 0.074 & -6.602 \\
4 & -3.766 & -3.671 & -0.343 & & 0.520 & 0.544 & 0.074 & -6.645 \\
5 & -2.531 & -2.465 & -0.231 & & 0.095 & 0.101 & 0.011 & -5.023 \\
6 & -2.131 & -2.079 & -0.196 & & -0.044 & -0.046 & -0.008 & -4.504 \\
\hline
\end{tabular}

Table S3. Parameters for the potential functions in equations (1) and (2).

\begin{tabular}{lrrrrrrr}
\hline state & $a$ & $D$ & $r_{e}$ & $V_{0}$ & $K$ & $\theta_{e}$ & $V_{0}^{\prime}$ \\
\hline 0 & 1.5576 & 0.11410 & 2.0946 & -0.57962 & 1.6992 & 105.17 & -0.57916 \\
1 & 1.4701 & 0.11502 & 2.1392 & -0.53609 & 1.9667 & 109.27 & -0.53636 \\
2 & 1.4497 & 0.11571 & 2.1760 & -0.52328 & 1.7597 & 109.34 & -0.52269 \\
3 & 1.5053 & 0.10706 & 2.1724 & -0.52274 & 1.9440 & 109.03 & -0.52263 \\
4 & 1.5023 & 0.10555 & 2.1882 & -0.51713 & 1.5762 & 110.05 & -0.51682 \\
5 & 2.3144 & 0.00103 & 2.8061 & -0.54868 & 1.2942 & 117.71 & -0.53747 \\
6 & 2.3260 & 0.00209 & 2.6827 & -0.51352 & 1.8569 & 109.57 & -0.50121 \\
\hline
\end{tabular}

$a$ is in $\AA^{-1}, r_{e}$ is in $\AA$.

$D, V_{0}$ and $V_{0}^{\prime}$ are in hartree.

$K$ is in $10^{-5}$ hartree $\mathrm{deg}^{-2}, \theta_{e}$ is in deg. 


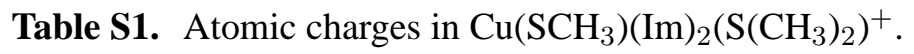

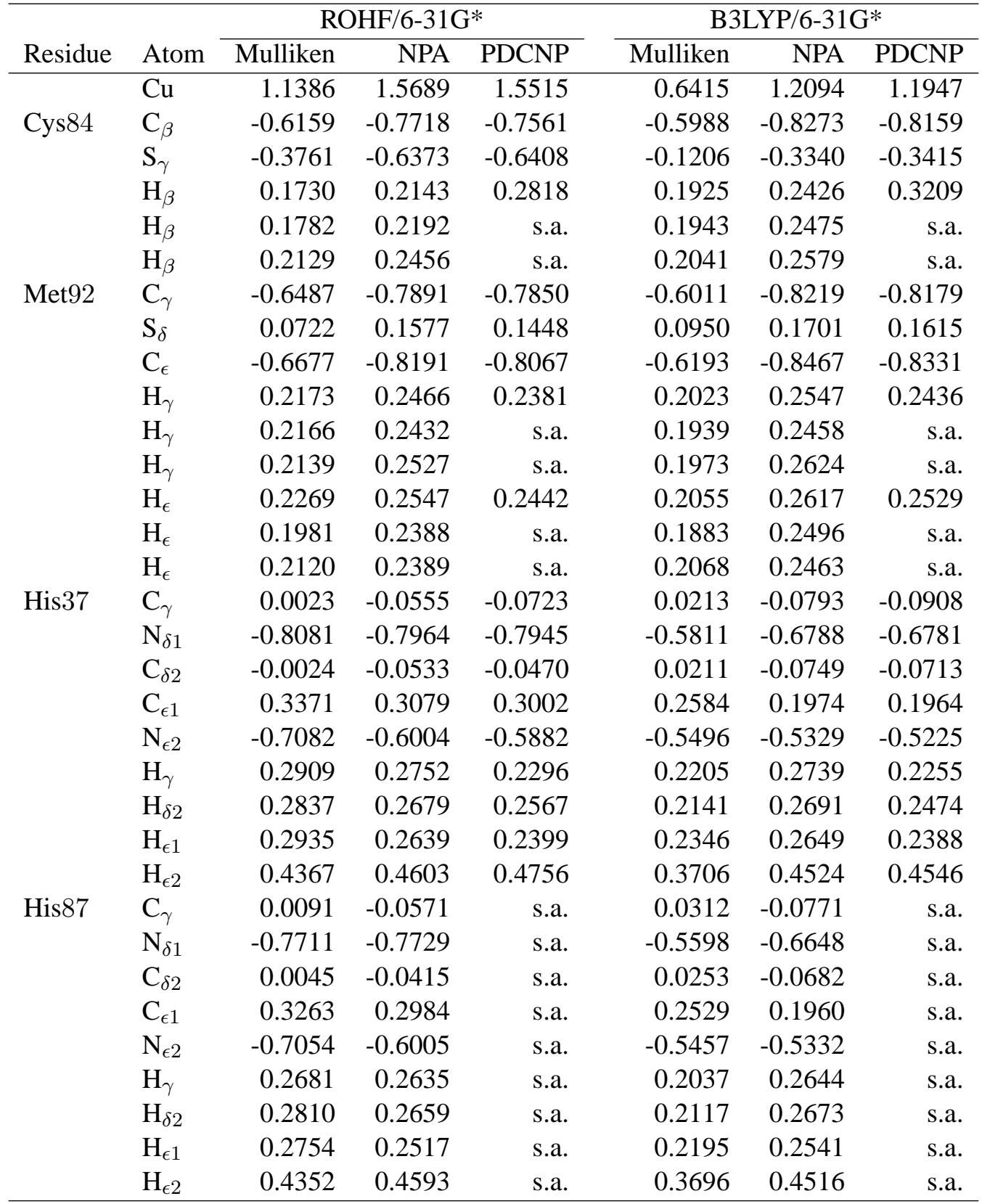

NPA = Natural Populations Analysis.

PDCNP $=$ Potential Derived Charges constrained around Natural Populations.

s.a. = same as above for the same atom type. 
Figure S1. Important active MOs and configurations from MCSCF-ORMAS calculation on the $\mathrm{Cu}\left(\mathrm{SCH}_{3}\right)^{+} / /\left(\mathrm{S}\left(\mathrm{CH}_{3}\right)_{2}\right)(\mathrm{Im})_{2}$ model.

\begin{tabular}{llllll} 
& & & \\
MO \#1 & & & & \\
\hline
\end{tabular}


Figure S2. Same as Figure $\mathrm{S} 1$ but for the $\mathrm{Cu}\left(\mathrm{SCH}_{3}\right)(\mathrm{Im})^{+} / /\left(\mathrm{S}\left(\mathrm{CH}_{3}\right)_{2}\right)(\mathrm{Im})$ model.
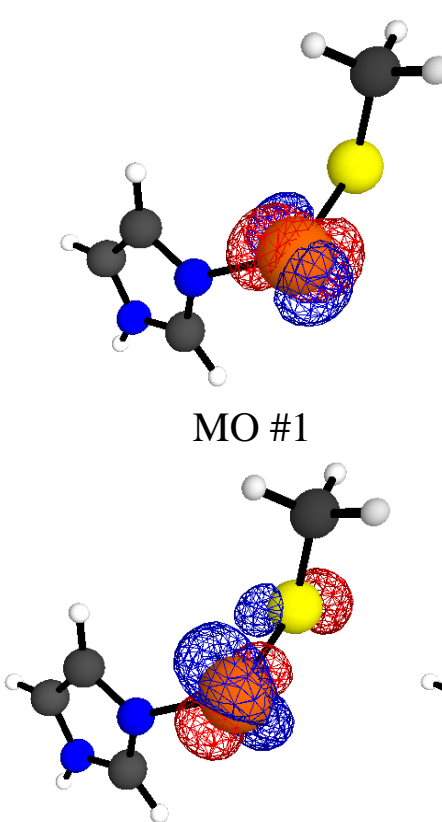

MO \#5

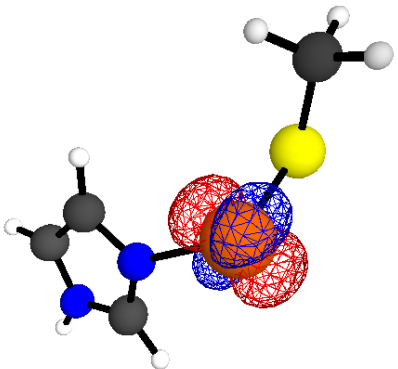

MO \#2

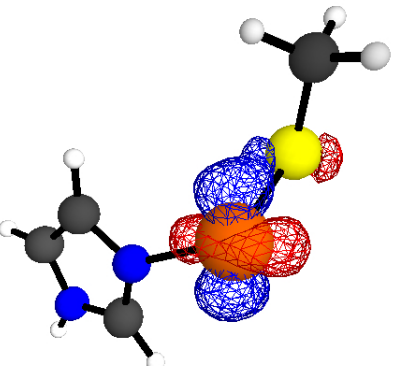

MO \#3

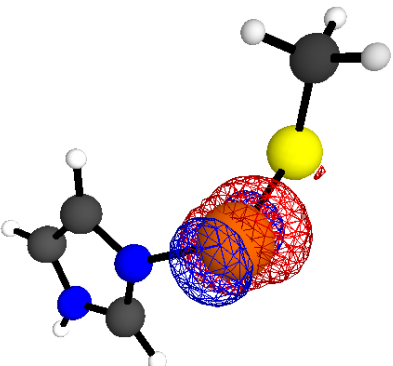

MO \#4

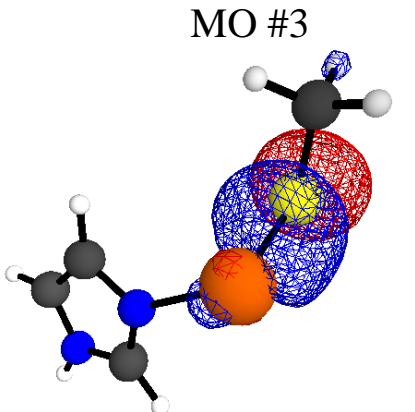

MO \#7

\begin{tabular}{llrrr}
\hline State & $\begin{array}{l}\text { Absolute } \\
\text { energy (a.u.) }\end{array}$ & $\begin{array}{l}\text { Excitation } \\
\text { energy }\left(\mathrm{cm}^{-1}\right)\end{array}$ & $\begin{array}{r}\text { Configuration } \\
\text { (Coefficient) }\end{array}$ \\
\hline $0(\mathrm{GR})$ & -2300.60935 & 0 & 2222122 & $(0.7419)$ \\
& & & 1222222 & $(0.3828)$ \\
& & & 2222212 & $(0.3461)$ \\
1 & -2300.59622 & 2880 & 1222222 & $(-0.3022)$ \\
& & & 2222122 & $(-0.7529)$ \\
& & & 2222221 & $(-0.3371)$ \\
$2(\mathrm{LF})$ & -2300.57398 & 7760 & 2122222 & $(0.6430)$ \\
& & & 2221222 & $(-0.5388)$ \\
& & & 2212222 & $(0.4855)$ \\
$3(\mathrm{LF})$ & -2300.57243 & 8100 & 2221222 & $(0.7711)$ \\
& & & 2122222 & $(0.4016)$ \\
& & & 2212222 & $(0.3766)$ \\
$4(\mathrm{LF})$ & -2300.57230 & 8130 & 2212222 & $(0.7590)$ \\
& & & 2122222 & $(-0.6120)$ \\
$5(\mathrm{CT})$ & -2300.52976 & 17470 & 222221 & $(0.7014)$ \\
& & & 122222 & $(0.4426)$ \\
$6(\mathrm{CT})$ & -2300.50632 & 22610 & 2222212 & $(0.7202)$ \\
& & & 2222122 & $(-0.3843)$ \\
\hline
\end{tabular}

The results for the model with the other His ligand included in the quantum part are qualitatively similar, with the excitation energies; $3120,7490,7700,8070,17180$, and $22350 \mathrm{~cm}^{-1}$. 
Table S4. Optimized coordinates (in $\AA$ ) of hydrogens from the ROHF/PM3 calculations, with the heavy atoms from the crystal structure (1bxu.pdb), as displayed in Figure 1.

\begin{tabular}{|c|c|c|c|c|}
\hline Residue & Atom & $\mathrm{X}$ & $\mathrm{Y}$ & $\mathrm{Z}$ \\
\hline & $\mathrm{Cu}$ & 0.00000 & 0.00000 & 0.00000 \\
\hline \multirow[t]{5}{*}{ Cys84 } & $\mathrm{S}_{\gamma}$ & 2.14179 & 0.00000 & 0.00000 \\
\hline & $\mathrm{C}_{\beta}$ & 2.64325 & 1.73794 & 0.00000 \\
\hline & $\mathrm{H}_{\beta}$ & 2.17260 & 2.33160 & -0.79150 \\
\hline & $\mathrm{H}_{\beta}$ & 2.44963 & 2.23948 & 0.95480 \\
\hline & $\mathrm{H}_{\beta}$ & 3.72870 & 1.75383 & -0.16899 \\
\hline \multirow[t]{9}{*}{ His37 } & $\mathrm{C}_{\gamma}$ & -1.02612 & -0.74706 & 2.73289 \\
\hline & $\mathrm{N}_{\delta}$ & -1.29468 & -0.28594 & 1.46136 \\
\hline & $\mathrm{C}_{\delta}$ & -2.21700 & -0.83650 & 3.36455 \\
\hline & $\mathrm{C}_{\epsilon}$ & -2.59303 & -0.12559 & 1.28894 \\
\hline & $\mathrm{N}_{\epsilon}$ & -3.18220 & -0.42195 & 2.44544 \\
\hline & $\mathrm{H}_{\gamma}$ & -0.00961 & -0.96654 & 3.08227 \\
\hline & $\mathrm{H}_{\delta}$ & -2.45031 & -1.15464 & 4.38649 \\
\hline & $\mathrm{H}_{\epsilon}$ & -3.08267 & 0.24219 & 0.37292 \\
\hline & $\mathrm{H}_{\epsilon}$ & -4.15368 & -0.38849 & 2.63036 \\
\hline \multirow[t]{9}{*}{ His87 } & $\mathrm{C}_{\gamma}$ & -0.89259 & -0.14960 & -2.86046 \\
\hline & $\mathrm{N}_{\delta}$ & -1.04563 & -0.63741 & -1.58796 \\
\hline & $\mathrm{C}_{\delta}$ & -1.58593 & -1.00060 & -3.68029 \\
\hline & $\mathrm{C}_{\epsilon}$ & -1.78097 & -1.71670 & -1.58511 \\
\hline & $\mathbf{N}_{\epsilon}$ & -2.12662 & -1.96944 & -2.86055 \\
\hline & $\mathrm{H}_{\gamma}$ & -0.29830 & 0.73777 & -3.09993 \\
\hline & $\mathrm{H}_{\delta}$ & -1.71050 & -0.97305 & -4.76765 \\
\hline & $\mathrm{H}_{\epsilon}$ & -2.03512 & -2.33123 & -0.70910 \\
\hline & $\mathrm{H}_{\epsilon}$ & -2.67340 & -2.73325 & -3.17059 \\
\hline \multirow[t]{9}{*}{ Met92 } & $\mathrm{C}_{\gamma}$ & -0.11097 & 4.37729 & -0.36771 \\
\hline & $\mathrm{S}_{\delta}$ & -0.89158 & 2.79657 & 0.03254 \\
\hline & $\mathrm{C}_{\epsilon}$ & -0.97005 & 2.99900 & 1.80387 \\
\hline & $\mathrm{H}_{\gamma}$ & -0.68832 & 5.21274 & 0.04712 \\
\hline & $\mathrm{H}_{\gamma}$ & 0.91998 & 4.45405 & -0.00059 \\
\hline & $\mathrm{H}_{\gamma}$ & -0.08840 & 4.49452 & -1.45857 \\
\hline & $\mathrm{H}_{\epsilon}$ & -0.61630 & 3.98441 & 2.14233 \\
\hline & $\mathrm{H}_{\epsilon}$ & -2.00427 & 2.90441 & 2.16043 \\
\hline & $\mathrm{H}_{\epsilon}$ & -0.36379 & 2.25221 & 2.33462 \\
\hline
\end{tabular}

